\title{
Inclusion Body Myositis Treated with Alemtuzumab
}

\author{
Juliana Sá ${ }^{1}$, João Costelha², Antonio Marinho ${ }^{3}$ \\ ${ }^{1}$ Centro Hospitalar Cova da Beira, Covilhã, Portugal \\ ${ }^{2}$ Unidade Local de Saúde do Alto Minho, EPE, Portugal \\ ${ }^{3}$ Centro Hospitalar do Porto, Porto, Portugal
}

\section{ABSTRACT}

Inclusion body myositis (IBM) is a chronic inflammatory myopathy with a progressive course. It is more common in the later years of life and usually presents with limb weakness. We present the case of a patient who developed proximal weakness in the lower limbs and, four years later, facial asymmetry. Blood analysis revealed high lactate dehydrogenase and creatinine kinase values. The diagnosis was obtained through muscle biopsy which met the histological criteria for IBM. The patient started treatment with alemtuzumab, leading to stabilisation of the symptoms in two years.

\section{LEARNING POINTS}

- IBM should be considered in the differential diagnosis of muscle weakness.

- Patients may present uncommon symptoms, such as prominent facial involvement.

- Alemtuzumab may potentially be beneficial in limiting the progression of IBM.

\section{KEYWORDS}

Inclusion body myositis, alemtuzumab, myopathy, treatment

\section{CASE PRESENTATION}

A 55-year-old female patient developed, seven years earlier, proximal weakness in the lower limbs, especially the left leg, with difficulty climbing stairs, albeit without much impact on her daily routines. Four years later, she developed facial asymmetry with right-side paresis, which was noticed by her relatives, associated with weakness in the left arm and first finger flexion (she scored 4/5 on the Medical Research Council Scale). The patient did not have any other symptoms, including dysphagia. There was no personal history of disease and no family history of muscle diseases. She had seven siblings, all without any known disease. Blood analysis, including blood count, renal and liver function, antinuclear antibodies and anti-acetylcholine antibodies, was negative or normal. Creatinine kinase (CK) values were initially in normal range, but peaked at $506 \mathrm{U} / \mathrm{L}$ four years later. EMG studies showed no abnormalities. Muscle biopsy of the left deltoid muscle was inconclusive due to fatty infiltration and tissue degeneration. Genetic testing for limb-girdle muscular dystrophy was negative. Inclusion body myositis (IBM) or facioscapulohumeral muscular dystrophy (FSHD) were thus considered the most likely diagnoses, however, FSHD genetic tests were negative. The patient initiated corticosteroid therapy (1 mg/kg of corticosteroid, tapered down to $0.5 \mathrm{mg}$ ) without any clinical benefit. One year later, azathioprine $25 \mathrm{mg}$ was added and tapered up to $100 \mathrm{mg}$ without clinical improvement. Ten years after the first symptoms, the patient presented at the outpatient clinic with a worsening of the weakness, which involved both legs, the left arm and face, and an episode of faecal incontinence. The patient was unable to get up from the floor and had to use her left hand for daily activities. At this point, she showed normal blood count and sedimentation velocity, lactate dehydrogenase of $276 \mathrm{U} / \mathrm{L}$ and $\mathrm{CK}$ of 345 
U/L. Renal function was normal, while hepatic enzymes were elevated and bilirubin was slightly elevated, the latter a finding associated with the azathioprine treatment. An MRI examination was performed in order to identify a muscle for a repeat muscle biopsy. The vastus lateralis muscle was biopsied and found to present adipose tissue substitution, some "rimmed vacuoles", and positivity for p62 and TDP43 on immunohistochemistry analysis. The patient thus met the histological criteria for IBM. She started alemtuzumab, 12 mg subcutaneously. One year after the beginning of the therapy the patient's symptoms had stabilised and she was able to rise from a chair and climb stairs. There was also a downgrading of $\mathrm{CK}$ to $153 \mathrm{U} / \mathrm{L}$. Follow up of the patient two years after the beginning of the treatment showed stabilisation of the clinical symptoms and she continues to be monitored.

\section{DISCUSSION}

Inclusion body myositis (IBM) is a chronic, slowly progressive inflammatory myopathy that is associated with muscle weakness. It is more common in the later years of life and usually presents with limb weakness. One of the most frequent symptoms is dysphagia; the facial and ocular muscles are frequently spared, however there are occasional reports of middle facial involvement. Biopsy is important for diagnosis, and histological features include the presence of multifocal myofibre-surrounding lymphocytic infiltrates and "rimmed vacuoles". It still remains to be understood whether the myofibre damage is due to primary degeneration or the inflammatory process ${ }^{[1]}$. Treatment is often ineffective and immunosuppressive treatment has little impact on the disease progression. Recent studies have reported some clinical benefits of alemtuzumab, a monoclonal antibody directed at CD52 that depletes lymphocytes, in IBM patients. IBM is more frequent in males and is usually associated with asymmetric muscle weakness ${ }^{[2]}$. Symptoms frequently involve the finger flexors and knee extensors, and dysphasia is present in $40 \%$ of patients. In this patient, the most prominent symptom was facial paresis. To our knowledge, there is only one other case in the literature of IBM with prominent facial involvement ${ }^{[1,2]}$. IBM is an inflammatory myopathy mediated by cytotoxic CD8+ T cells that clonally expand in situ and invade MHC-I-expressing muscle fibres. As regards its treatment, one of the clinical features of IBM is the poor effectiveness of corticosteroids and immunosuppressants in improving symptoms, as also seen in this patient. Alemtuzumab is a monoclonal antibody that destroys blood cells with CD52 surface protein; it has been used in autoimmune diseases and in small samples of patients with IBM ${ }^{[3,4]}$. Trials are ongoing to better understand the pathogenesis and treatment of IBM. However, it should be considered in the differential diagnosis of muscle weakness, even in patients, like the case presented, with an uncommon clinical presentation of the disease. In the present case, symptoms were stabilised after the beginning of therapy with alemtuzumab, and the patient even showed a slight improvement in her ability to perform daily living activities, such as climbing stairs. This case may constitute additional evidence of the potential benefit of alemtuzumab in limiting the progression of IBM, however, further investigation is needed.

\section{REFERENCES}

1. Lam L, Scheper S, Zagorski N, Chung M, Noguchi H, Liow KK. Inclusion body myositis: a case of bilateral extremity weakness. Hawaii J Med Public Health 2013;72:417-420.

2. Suwa Y, Suzuki N, Soga T, et al. Sporadic inclusion body myositis manifesting as isolated muscle weakness of the finger flexors three years after disease onset. Intern Med 2016;55:3521-3524.

3. Schmit K, Kleinschinitz K, Rakocevi G, Dalakas MC, Schmidt J. Molecular treatment effects of alemtuzumab in skeletal muscles of patients with IBM. BMC Neurology 2016;16:48.

4. Dalakas MC, Rakocevic G, Schmidt J, Salajegheh M, McElroy B et al. Effect of alemtuzumab (CAMPATH 1-H) in patients with inclusion-body myositis. Brain 2009;132:15361544. 